\title{
Analogy between Conventional Grid Control and Islanded Microgrid Control based on a Global dc-Link Voltage Droop
}

\author{
T. L. Vandoorn, B. Meersman, J. D. M. De Kooning and L. Vandevelde
}

\begin{abstract}
For islanded microgrids, droop-based control methods are often used to achieve a reliable energy supply. However, in case of resistive microgrids, these control strategies can be rather different to what conventional grid control is accustomed to. Therefore in this paper, the theoretical analogy between conventional grid control by means of synchronous generators (SGs) and the control of converter-interfaced distributed generation (CIDG) units in microgrids is studied. The conventional grid control is based on the frequency as a global parameter showing differences between mechanical power and ac power. The SGs act on changes of frequency through their $P / f$ droop controller, without inter-unit communication. For CIDG units, a difference between dc-side power and ac-side power is visible in the dc-link voltage of each unit. Opposed to grid frequency, this is not a global parameter. Thus, in order to make a theoretical analogy, a global measure of the dc-link voltages is required. A control strategy based on this global voltage is presented and the analogy with the conventional grid control is studied, with the emphasis on the need for interunit communication to achieve this analogy. A known control strategy in resistive microgrids, called the voltage-based droop control for CIDG units, approximates this analogy closely, but avoids inter-unit communication. Therefore, this control strategy is straightforward for implementation as it is close to what control engineers are used to. Also, it has some specific advantages for the integration of renewables in the network.
\end{abstract}

Index Terms-microgrid, distributed generation, droop control, conventional grid control, voltage-source inverter

\section{INTRODUCTION}

With the advent of large amounts of distributed generation (DG) units, the power system undergoes major changes, especially at the distribution level [1]-[3]. Therefore, the microgrid concept has been developed [4]-[6]. Microgrids enable a coordinated integration of the DG units in the electrical power system and capture the emerging potential of DG [7]. Opposed to the SGs, a high share of the DG units are not directly coupled to the electrical network, but use powerelectronic converter interfaces [8]. These converter-interfaced DG (CIDG) units lack the rotating inertia the conventional grid control is based on. Also, islanded microgrids have very different characteristics in comparison with the conventional electrical system, such as their small scale and the possible high share of renewable and volatile energy sources. Therefore, for islanded microgrids, new control strategies for these CIDG units have been developed. In order to avoid single

The authors are with the Dept. of Electrical Energy, Systems \& Automation, Ghent University, St-Pietersnieuwstraat 41, 9000 Gent, Belgium, e-mail Tine.Vandoorn@UGent.be. The work of T. Vandoorn is financially supported by a Fellowship of the FWO-Vlaanderen (Research Foundation - Flanders, Belgium). The research was carried out in the frame of the Inter-university Attraction Poles programme IAP-VI-021, funded by the Belgian Government. The research of J. D. M. De Kooning is funded by the Special Research Fund (BOF) of Ghent University (Belgium). points of failure and to increase the reliability of the microgrid, the usage of communication for the primary control is often avoided. This has led to the development of droop-based control strategies.

The $P / f$ droop control [9]-[11], with many variants, is widely used as it is based on the conventional grid control. In [11], both single master operation (one unit with droop control) and multi-master operation (multiple units with droop control) are considered, in this paper we focus on multi-master operation with multiple units taking part in the power sharing and balancing of the network. The $P / f$ droop control strategy is based on the inductive character of the lines. However, as many microgrids are low-voltage networks, the lines are often mainly resistive. Therefore, the so-called reversed droops, $P / V_{\mathrm{g}}$ droops, have been developed [12], [13]. A variant of this strategy, the voltage-based droop (VBD) control in [14], combines $P / V$ droop control with dc-link voltage droops. In the VBD control strategy, the power changes of renewable energy sources can easily be postponed (to a higher difference between the terminal voltage and its nominal value) compared to that of the dispatchable DG units. This is achieved through the usage of constant-power bands, which is clarified in $\S$ III-B. This can lead to an optimized integration of renewable energy sources in the system. Another modification on the droop control strategy is to use a control loop known as the virtual output impedance loop to fix the output impedance of the inverter, to increase the stability of the system and to share linear and nonlinear loads [15], [16]. In case of VBD control, a resistive output impedance is chosen as this provides more damping in the system [17], [18] and complies with the power control strategies of the loads and generators, where the active power is changed based on the grid voltage.

In this paper, the theoretical analogy between conventional grid control by means of SGs and resistive islanded microgrid control through CIDG units is studied. There is an analogy between the rotating inertia of SGs and the dc-link capacitor of DG units, as they form the storage capacity for transient active power changes. A second analogy is present between the grid frequency in case of SGs and the dc-link voltage of DG units, as they show the state of the network. A changing grid frequency/dc-link voltage indicates a difference between the input power and the ac power delivered to the network. Therefore, a control strategy, called global dc-link voltagebased droop (GVBD) control, is presented that uses the dclink voltage as control parameter for the CIDG units analogous to the grid frequency for SGs. Opposed to the frequency, the dc-link voltage is not a global parameter. Therefore, in order to make the theoretical analogy complete, communication is required to determine a global parameter presenting the dc-link voltage of all DG units, the so-called global dc-link voltage. 
TABLE I

NOMENCLATURE

$\begin{array}{ll}b & \text { Constant-power band width (p.u.) } \\ C_{\mathrm{dc}} & \text { Dc-link capacitance }(\mathrm{F}) \\ C E & \text { Capacitor energy } \\ C_{f} & \text { Filter capacitance }(\mathrm{F}) \\ \mathrm{CIDG} & \text { Converter-interfaced DG } \\ \mathrm{CPFD} & \text { Conventional } P / f \text { droop } \\ \mathrm{DG} & \text { Distributed generation } \\ f & \text { Grid frequency }(\mathrm{Hz}) \\ \mathrm{GVBD} & \text { Global voltage-based droop } \\ I_{\mathrm{dc}} & \text { Dc-side current }\end{array}$

This GVBD control follows the theoretical analogy with conventional grid control, but requires inter-unit communication. In this paper, it is shown that the VBD control, which does not require inter-unit communication, lies very close to this global dc-link voltage control. Therefore, the VBD control in resistive networks is very similar to the conventional grid control, here referred to as conventional $P / f$ droop (CPFD) control. By using this VBD control strategy, an operation of the resistive microgrid that lies very close to what engineers are used to in conventional networks can be obtained.

This paper is structured as follows. First, the conventional grid control with $P / f$ droops is briefly discussed. Second, the islanded microgrid control with the $P / f$ and the reversed droop control strategies is summarized, highlighting the VBD controller. Third, the analogy between conventional grid control and islanded microgrid control is made by including a global dc-link voltage and using it in a GVBD controller. Finally, some cases are studied to verify the GVBD control strategy and to compare it with CPFD control and VBD control. Both a basic network and a more realistic microgrid are studied.

\section{CONVENTIONAL GRID CONTROL}

The conventional grid control, for large centralised SGs connected to the transmission network, is largely based on the rotating inertia of the network. In case of a difference between the mechanical input power $P_{m}$ and the electrical output power $P$ in Fig. 1, the rotational speed of the generator will change because of the presence of rotating inertia $J$. The grid frequency $f$ is directly coupled with the rotational speed. Therefore, in case of a load change in the network, the SGs will all measure a changed grid frequency, as frequency is a global parameter. The CPFD control strategy of the SGs is designed such that the prime movers react on the frequency changes by means of a droop control mechanism:

$$
P_{\mathrm{m}}=P_{\mathrm{m}, \mathrm{nom}}-K_{P}\left(f-f_{\mathrm{nom}}\right)
$$

with $K_{P}$ the droop coefficient or statism of the generator. $K_{P}$ is tuned according to the type of energy source and the ratings of the SGs. The CPFD control provides power balancing and power sharing between the units according to their ratings, is responsible for the fast primary control and does not require communication.

\section{CONTROL OF CIDG UNITS IN AN ISLANDED MICROGRID}

This paper focuses on droop-based control for the active power sharing and voltage regulation in an islanded microgrid.

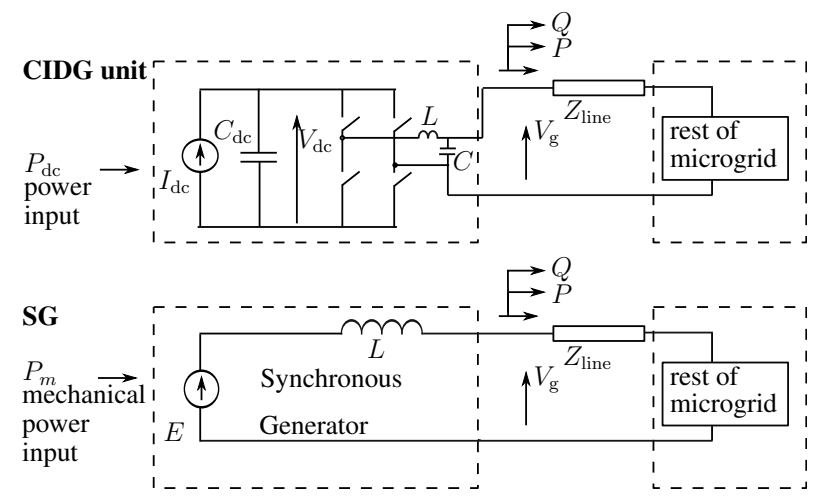

Fig. 1. Synchronous generator (SG) versus voltage-source inverter (VSI) in case of a converter-interfaced DG (CIDG) unit

A single-phase system is studied in this paper. In threephase systems, the control principle remains analogous, the implementation in three-phase systems is out of the scope of this paper. Islanded microgrids are generally fed with CIDG units (see Fig. 1). These units generally have no rotating inertia or are not directly coupled to the grid (thus, the rotational speed of the generator is not directly coupled with the grid frequency), just like the loads. Therefore, the islanded microgrids lack the inertia the conventional grid control is largely based on.

\section{A. Droop control based on conventional grid control}

In literature, droop controllers based on the conventional grid control are often used. For the active power sharing between multiple DG units, the $P / f$ droop control strategy:

$$
f=f_{\text {nom }}-K_{\mathrm{p}}\left(P-P_{\text {nom }}\right)
$$

with $K_{\mathrm{p}}$ the droop coefficient, can be implemented [9], [13], [19]-[22]. This control strategy is based on the linkage between the active power and the phase angle in a network with inductive line parameters. The phase angle is dynamically determined by the grid frequency. Theoretically, this control does not require inertia in the system, as it is based on the power flow characteristics of inductive lines. Therefore, the droop can be based on $f(P)$ as in 2 or reversely, $P(f)$, where the frequency is measured and drooped to determine $P$. In contrast with measurements of power, accurate measurements of frequency are not straightforward. Therefore, often the $f(P)$ strategy is chosen [13]. 
Different variants and additions have been implemented in the $P / f$ droop control method. To avoid line impedance influence on the power sharing and to improve decoupling between $P$ and $Q$, a virtual output impedance control loop is included in the traditional droop method [18], [23], [24]. To improve the dynamic performance of paralleled inverters, in [10], derivative-integral terms are introduced into the conventional droop scheme and a soft-start operation of the output impedance is proposed to alleviate the initial transient peak.

\section{B. Reversed droops}

In the islanded microgrids connected to the low-voltage distribution network that are considered in this paper, the $P / f$ droop control strategy poses some problems, due to the lack of rotating inertia and the resistive microgrid lines. An increasing share of the network elements are converter-interfaced, such that the considered microgrids lack a significant rotating inertia in the generators and loads. However, it would still be possible to use $P / f$ droops, if the network lines were predominantly inductive. In this case, the $P / f$ droops are based on a coupling between $P$ and $f$, which is present in inductive networks with inertia. However, the considered small-scale microgrids are connected to the low-voltage distribution network which can be assumed as mainly resistive. This is a valid assumption because of the high $\mathrm{R} / \mathrm{X}$ values, e.g., a typical $\mathrm{R} / \mathrm{X}$ value in lowvoltage lines is 7.7 [13], [25]. Also, by implementing resistive virtual output impedances in the inverters, the resistive nature of the network increases even more. In the resistive networks, there is mainly a linkage between the active power and the voltage instead of the phase angle. Therefore, the reversed $P / V_{\mathrm{g}}$ droop control strategy is developed in [12], [13], [26], [27]:

$$
P=P_{\text {nom }}-K_{V}\left(V_{\mathrm{g}}-V_{\mathrm{g}, \text { nom }}\right)
$$

with $K_{V}$ the droop and $V_{\mathrm{g}}$ the rms grid voltage. To further improve the steady-state and transient response of the reversed droops, resistive output impedance is included in the control strategy, which allows good power sharing with low sensitivity to the line impedance [15].

A variant of reversed droop control is the combination of $V_{\mathrm{g}} / V_{\mathrm{dc}}$ and $P / V_{\mathrm{g}}$ droop control proposed in [14], with $V_{\mathrm{dc}}$ the dc-link voltage. This so-called VBD control strategy is based on the lack of inertia, the mainly resistive line parameters and the high share of renewable energy sources in low-voltage islanded microgrids. Because of the usage of power-electronic interfaces, a difference between the input dc-side power $P_{\mathrm{dc}}$ and the ac-side power $P$ is visible in the dc-link voltage $V_{\mathrm{dc}}$. Therefore, the voltage droops are realized by the $V_{\mathrm{g}} / V_{\mathrm{dc}}$ droop controller, which changes the rms microgrid voltage in response to changes of the dc-link voltage:

$$
V_{\mathrm{g}}=V_{\mathrm{g}, \text { nom }}+K_{\mathrm{a}}\left(V_{\mathrm{dc}}-V_{\mathrm{dc}, \text { nom }}\right)
$$

Even a slight change of $V_{\mathrm{g}}$ leads to a change of the power delivered to the electrical network [14]. This effect is realised by a natural balancing due to resistive loads and microgrid lines. As the voltage in a microgrid is allowed to vary between certain limits [28], the tolerable voltage band can be actively exploited for the power control. With the $V_{\mathrm{g}} / V_{\mathrm{dc}}$ droop controller solely, the input power is not controlled. If a certain voltage level $\left((1 \pm b / 2) V_{\mathrm{g}, \text { nom }}\right)$ is exceeded, also the input power of the DG unit is changed by means of $P / V_{\mathrm{g}}$ droop control (depicted in Fig 2) to avoid voltage limit violation:

$$
P_{\mathrm{dc}}=\left\{\begin{array}{l}
P_{\mathrm{dc}, \text { nom }}-K_{\mathrm{V}}\left(V_{\mathrm{g}}-(1+b / 2) V_{\mathrm{g}, \text { nom }}\right) \\
\quad \text { if } V_{\mathrm{g}}>(1+b / 2) V_{\mathrm{g}, \text { nom }} \\
P_{\mathrm{dc}, \text { nom }} \\
\quad \text { if }(1-b / 2) V_{\mathrm{g}, \text { nom }}<V_{\mathrm{g}}<(1+b / 2) V_{\mathrm{g}, \text { nom }} \\
P_{\mathrm{dc}, \text { nom }}-K_{\mathrm{V}}\left(V_{\mathrm{g}}-(1-b / 2) V_{\mathrm{g}, \text { nom }}\right) \\
\quad \text { if } V_{\mathrm{g}}<(1-b / 2) V_{\mathrm{g}, \text { nom }}
\end{array}\right.
$$

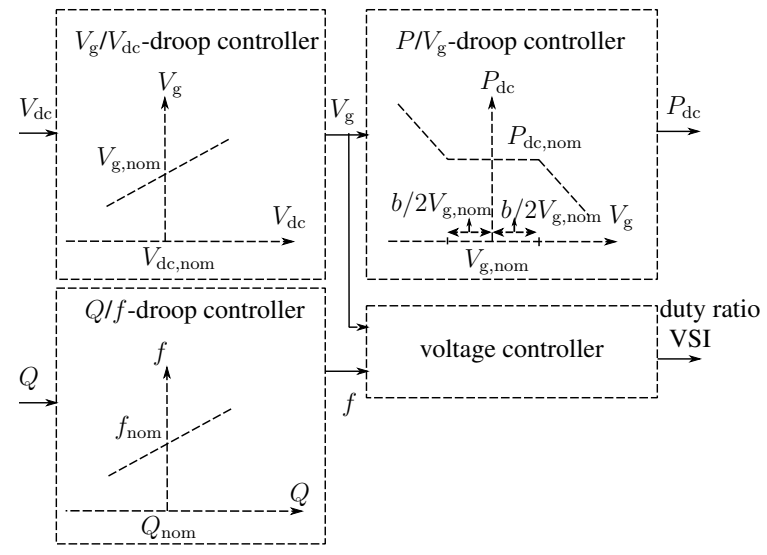

Fig. 2. Combined operation of the droop controllers to determine the set value of the grid voltage in case of VBD control

The combination of these two droops is a further improvement of the $P / V_{\mathrm{g}}$ droop control concept. This control strategy leads to constant power output in a voltage band with a width $b$ depending on the nature of the power source. A wide constant-power band is implemented in the renewable sources, as depicted in Fig. 3 . Hence, the power changes of the renewables can be postponed to more extreme voltage deviations from the nominal voltage, compared to those of the dispatchable DG units. Hence, in a voltage band around the nominal voltage, the renewable DG units operate at their optimal operating point, while their power change in case of extreme voltages can still be coordinated without inter-unit communication. In this way, a more efficient usage of the renewable energy can be achieved. This can even lead to an increased share of renewables in islanded microgrids.

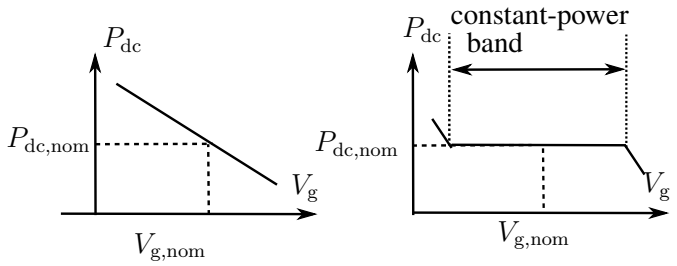

a) dispatchable unit

b) less controllable unit

Fig. 3. Constant-power band of fully dispatchable versus less controllable DG units

In [17], this VBD control strategy is combined with an active load control based on the same principles to allow reliable power supply and load response without inter-unit communication for the robust primary control. 
For the reactive power sharing, $Q / f$ droop control is used:

$$
f=f_{\text {nom }}+K_{Q}\left(Q-Q_{\text {nom }}\right)
$$

with $K_{Q}$ the droop; mostly, $Q_{\text {nom }}=0$ VAr. The phase angle of the reference voltage is dynamically determined by $f$.

\section{AnAlOgY CONVENTIONAL GRID CONTROL/MICROGRID CONTROL}

\section{A. Parameter for active power change}

In the conventional grid control, the grid frequency is a global parameter that shows the state of the network. In an islanded low-voltage microgrid (thus, with resistive line parameters and lack of inertia), no such global parameter exists. The active power is linked with the grid voltage, but this is not a global parameter because of the voltage drops over the lines.

A change of consumption or generation instantly affects the dc-link voltages of the DG units, because of the presence of dc-link capacitors. If the generated dc-power of the unit $P_{\mathrm{dc}}$ is higher than the ac-power $P$, the dc-link voltage will increase, and vice versa for $P_{\mathrm{dc}}<P$. This is analogous to the change of grid frequency because of the presence of rotating inertia in conventional networks. If the CIDG units are controlled by control strategies that keep the dc-link voltage equal to a predefined value (e.g. proportional-integral controller), only the transient state is visible in $V_{\mathrm{dc}}$. However, in this paper, with the $V_{\mathrm{g}} / V_{\mathrm{dc}}$ droop controller that controls $V_{\mathrm{dc}}$ to a constant but not necessary predefined value (proportional controller), $V_{\mathrm{dc}}$ shows the overall system state. High $V_{\mathrm{dc}}$ indicates a low load burden on the unit, while a low dc-link voltage is present in case of a heavily loaded DG unit.

Still, opposed to the grid frequency, $V_{\mathrm{dc}}$ is not a global parameter, thus, for the analogy between conventional grid control and islanded microgrid control, a global parameter representing the state of all units is required. This global parameter can be obtained from the balancing energy. In the conventional system, the kinetic energy (KE) stored in the system equals:

$$
\mathrm{KE}=\frac{1}{2} J \omega^{2}
$$

with $J$ the total rotating inertia of the system and $\omega=2 \pi f$. Changes of $f$ show differences between $P_{\mathrm{m}}$ and $P$ that are balanced by a changed $\mathrm{KE}$ of the system. In the islanded microgrid, the balancing energy is obtained from the energy in the dc-link capacitors (capacitor energy $\mathrm{CE}$ ):

$$
\mathrm{CE}=\sum_{\mathrm{i}=1}^{\mathrm{N}} \frac{1}{2} C_{\mathrm{dc}, \mathrm{i}} V_{\mathrm{dc}_{\mathrm{i}}}^{2}
$$

with $\mathrm{N}$ the number of dc-link voltages. A complete analogous energy equation is obtained, except that opposed to $f$, the dc-link voltage is not a global parameter. Again, a difference between $P_{\mathrm{dc}}$ and $P$ is balanced by a changed CE.

Hence, a change of $\mathrm{CE}$ compared to the nominal value can be used as the required global parameter:

$$
e_{\mathrm{dc}, \mathrm{g}}=\frac{1}{N} \sum_{i=1}^{N} \frac{C_{\mathrm{dc}, \mathrm{i}} V_{\mathrm{dc}, \mathrm{i}}^{2}-C_{\mathrm{dc}, \text { nom }, \mathrm{i}} V_{\mathrm{dc}, \text { nom }, \mathrm{i}}^{2}}{C_{\mathrm{dc}, \text { nom }, \mathrm{i}} V_{\mathrm{dc}, \text { nom }, \mathrm{i}}^{2}}
$$

This is analogous to

$$
\frac{1}{N} \frac{J \omega^{2}-J \omega_{\text {nom }}^{2}}{J \omega_{\text {nom }}^{2}}
$$

from which $f$ can be calculated. Hence, $e_{\mathrm{dc}, \mathrm{g}}$ and $f$ can be used as parameters in $P / e_{\mathrm{dc}, \mathrm{g}}$ and the conventional $P / f$ droop controllers that are implemented in an analogous way.

In this paper, the units have equal nominal dc-link voltages and capacitors. Therefore, to limit the computational burden, also the parameter $v_{\mathrm{dc}, \mathrm{g}}$ can be used instead of $e_{\mathrm{dc}, \mathrm{g}}$ :

$$
v_{\mathrm{dc}, \mathrm{g}}=\frac{1}{N} \sum_{i=1}^{N} \frac{V_{\mathrm{dc}, \mathrm{i}}-V_{\mathrm{dc}, \mathrm{nom}, \mathrm{i}}}{V_{\mathrm{dc}, \mathrm{nom}, \mathrm{i}}}
$$

$v_{\mathrm{dc}, \mathrm{g}}$ is a dimensionless parameter that represents the global change of dc-link voltage. However, if the difference between the capacitance of the dc-link capacitors is significant, using $e_{\mathrm{dc}}$ instead of $v_{\mathrm{dc}, \mathrm{g}}$ is the logical approach to make a control in resistive microgrids that is analogous to the CPFD control in conventional networks.

\section{B. Droop controller based on global dc-link voltage}

The global $v_{\mathrm{dc}, \mathrm{g}}$ is then drooped, with a negative slope, to determine the dc-power of the unit as shown in Fig. 4.

$$
P_{\mathrm{dc}}=P_{\mathrm{dc}, \text { nom }}-K_{g} v_{\mathrm{dc}, \mathrm{g}}
$$

This droop is analogous to (1) by linking $f$ with $v_{\mathrm{dc}, \mathrm{g}}$. The droop $K_{g}$ can be tuned analogously to the statism of conventional generators. A low value of $K_{\mathrm{g}}$ is included in little-dispatchable units, while high $K_{g}$ are used in case of dispatchable units.

Note that in order to determine $V_{\mathrm{dc}}$ in a single-phase system, a sample rate of twice the fundamental grid frequency is required to filter the ripple in the dc-link voltage because of the full-bridge configuration of the voltage-source inverter (VSI). Therefore, for the communication of $v_{\mathrm{dc}, \mathrm{g}}$, an important advantage is that only low-bandwidth communication is required. Also, if this communication fails, instead of the global $v_{\mathrm{dc}, \mathrm{g}}$, the local $V_{\mathrm{dc}}$ can be used in the droop to determine $P_{\mathrm{dc}}$, while the synchronisation is performed locally through the $Q / f$ droop controller. This $Q / f$ droop controller is analogous as in the VBD control and is based on the linkage between $Q$ and $f$ in the considered low-voltage resistive microgrid [12], [15].

With the controller in (12) alone, $V_{\mathrm{g}}$ would remain constant. This would lead to an inadequate operation in the resistive network because in this case, the active power sharing is determined by the line impedances instead of the ratings of the DG units. Therefore, the $V_{\mathrm{g}} / V_{\mathrm{dc}}$ droop controller is used. In case of an excess of the dc-side power compared to the ac-side power of a DG unit, $V_{\mathrm{dc}}$ increases. In this case, the $V_{\mathrm{g}} / V_{\mathrm{dc}}$ droop controller increases $V_{\mathrm{g}}$. Even a slight change of $V_{\mathrm{g}}$ leads to a change of the power delivered to the electrical network by the inverter. This effect is realised by a natural balancing due to resistive loads and microgrid lines, and by intelligent loads that use voltage as trigger for the active load control [17]. The $P / v_{\mathrm{dc}, \mathrm{g}}$ droop controller will lower $P$ in case of increased $v_{\mathrm{dc}, \mathrm{g}}$, to avoid voltage limit violation and to achieve accurate power sharing and balancing.

The overall control schemes of both the GVBD and VBD control are summarized in Fig. 6. In conclusion, in the analogy 
for units $1, \cdots, N$

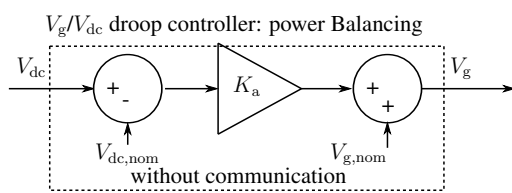

Central unit (signals from N DG units)

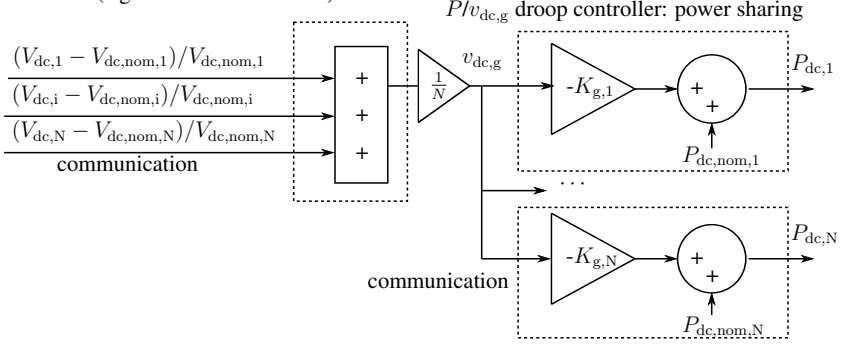

Fig. 4. GVBD control (here a dispatchable DG unit is considered: $b=0$ ), $v_{\mathrm{dc}, \mathrm{g}}$ is communicated to each CIDG unit and used in the $P / v_{\mathrm{dc}, \mathrm{g}}$ droop control. This controller cooperates with the $V_{\mathrm{g}} / V_{\mathrm{dc}}$ droop controller. In VBD control, the $V_{\mathrm{g}} / V_{\mathrm{dc}}$ and $P / V_{\mathrm{g}}$ droop controllers cooperate without inter-unit communication.

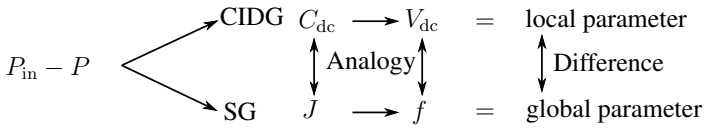

Fig. 5. Analogy between $J$ and $C_{\mathrm{dc}} ; V_{\mathrm{dc}}$ and $f$ ( $P_{\mathrm{in}}$ is the input power of the unit: $P_{\mathrm{in}}=P_{\mathrm{m}}$ in case of a SG, and $P_{\mathrm{in}}=P_{\mathrm{dc}}$ in case of a CIDG unit)
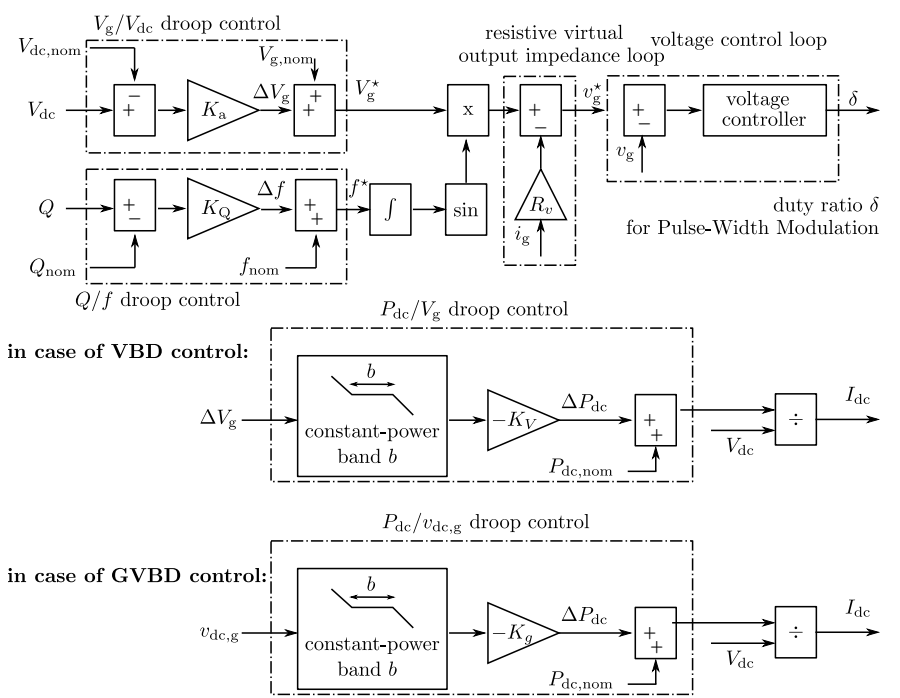

Fig. 6. Control algorithm of GVBD and VBD control

between GVBD control of CIDG units and CPFD control of SGs, the dc-link capacitor $C_{\mathrm{dc}}$ has the function of the rotating inertia $J$ in the conventional grid control and the global dclink voltage $v_{\mathrm{dc}, \mathrm{g}}$ functions as the grid frequency $f$ as depicted in Fig. 5 .

\section{VBD control as variant of GVBD control without inter-unit communication}

In SGs with CPFD control, $P$ is drooped with $f$, while in the analogy of CIDG units with GVBD control, $P$ is drooped with $v_{\text {dc,g. }}$. The VBD control strategy, consisting of $V_{\mathrm{g}} / V_{\mathrm{dc}}$ and $P / V_{\mathrm{g}}$ droop controllers, matches the GVBD control strategy very closely in resistive networks. The $P / V_{\mathrm{g}}$ droop controller in VBD control is analogous to the $P / v_{\mathrm{dc}, \mathrm{g}}$ droop controller in GVBD control because of the linear relationship between $V_{\mathrm{dc}}$ and $V_{\mathrm{g}}$ through the $V_{\mathrm{g}} / V_{\mathrm{dc}}$ droop controller. An exact match in the analogy between VBD control and CPFD control is not possible, opposed to the case of GVBD droop control, because the VBD control does not use interunit communication. Therefore, not the global $v_{\mathrm{dc}, \mathrm{g}}$ is known but only the local $V_{\mathrm{dc}}$ is visible for each DG unit separately.

The analogy is, thus, precise if the microgrid is fed by only one dispatchable unit $\left(v_{\mathrm{dc}, \mathrm{g}} V_{\mathrm{dc}, \mathrm{nom}}=V_{\mathrm{dc}}-V_{\mathrm{dc}, \text { nom }}\right)$. It is also precise in case of a combination of one dispatchable unit and several units operating in the constant-power band (with $\left.P_{\mathrm{dc}}=P_{\mathrm{dc}, \text { nom }}\right)$. In case of multiple dispatchable units, the analogy is approximately valid if it is considered in a local manner $\left(v_{\mathrm{dc}, \mathrm{g}} V_{\mathrm{dc}, \mathrm{nom}}=V_{\mathrm{dc}}-V_{\mathrm{dc}, \text { nom }}\right.$ locally). The local nature of the VBD control is one of its intrinsic advantages. In case of, e.g., high renewable generation and a low local load, the grid voltage will locally increase. This is because of the usage of constant-power bands such that the renewable energy sources will only change their power dependent on the state of the network in case of extreme voltages. Because of the local nature of these high voltages, only dispatchable DG units located electrically nearby will change their generated power. This can decrease the line losses and avoid congestion problems as locally consumed power is locally generated. It reflects the advantage of the local nature of the VBD control.

In conclusion, VBD control is similar to GVBD control, with the latter being completely analogous to conventional grid control. Therefore, this control strategy can be implemented in a manner close to what control engineers are used to. The local nature of VBD control, opposed to GVBD and CPFD control, has some important advantages, as discussed above.

\section{ISLANDED MICROGRIDS: CPFD CONTROL WITH SGS VERSUS GVBD AND VBD CONTROL WITH CIDG UNITS}

In this paragraph, first, a basic microgrid is studied consisting of two generators and two loads. The cases of GVBD control and CPFD control are compared in order to study the theoretical analogy between both control strategies. Next, the same case with VBD control is considered to prove that this control strategy follows the analogy with CPFD control closely, without need for inter-unit communication opposed to the GVBD control. Finally, the three controllers (GVBD, VBD and CPFD control) are studied in case of a more realistic microgrid with dynamic events and three generators.

\section{A. CIDG units with GVBD control}

In this first case, two DG units $G_{1}$ and $G_{2}$ are feeding a constant-power load $P_{\text {load, } 1}$ of 2 p.u. $\left(P_{\text {ref }}=1 \mathrm{~kW}\right)$ in islanded mode as depicted in Fig. 7 . After $1 \mathrm{~s}$, a second load of 1 p.u. turns on as well. The VSIs are modelled upto the level of the switches in a full-bridge configuration. The VSIs have an LC filter with $L=2 \mathrm{mH}$ and $C=3 \mu \mathrm{F}$, the nominal grid voltage equals 1 p.u. rms $\left(V_{\text {ref }}=230 \mathrm{~V}\right)$. The dc-link capacitances $C_{\mathrm{dc}}$ equal $1.5 \mathrm{mF}$ and the input dc-current $I_{\mathrm{dc}}$ equals $P_{\mathrm{dc}} / V_{\mathrm{dc}}$, with $P_{\mathrm{dc}}$ determined according to 12 and 
$V_{\mathrm{dc}}$ the dc-link voltage. The two DG units have nominal power $P_{1, \text { nom }}=0.9$ p.u. and $P_{2, \text { nom }}=1.2$ p.u. The line impedances are assumed as purely resistive and $Z_{\text {line }}=0.009$ p.u. and a resistive virtual output impedance $z_{v}=0.056$ p.u. of the VSI is included. Note, that the nominal voltage in (4) is adapted according to $z_{v}$ :

$$
V_{\mathrm{g}, \mathrm{nom}}=V_{\mathrm{ref}}+z_{v} \frac{P_{\mathrm{nom}}}{V_{\mathrm{ref}}}
$$

and $K_{\mathrm{a}}=\frac{0.5}{\sqrt{2}}$. The global measure of $V_{\mathrm{dc}}$ is determined according to [11), with $N=2$. This parameter $v_{\mathrm{dc}, \mathrm{g}}$ is then drooped according to the GVBD control strategy, with a negative slope $K_{g}$ to determine the dc-power of the unit. In the following simulations: $V_{\mathrm{dc}, \mathrm{nom}}=450 \mathrm{~V}, K_{\mathrm{g}, 1}=$ $100 V_{\mathrm{dc}, \text { nom }} \frac{2 P_{1, \text { nom }}}{P_{1, \text { nom }}+P_{2, \text { nom }}}$ and $K_{\mathrm{g}, 2}=K_{\mathrm{g}, 1} \frac{P_{2, \text { nom }}}{P_{1, \text { nom }}}$. For the reactive power control, which is not the focus of this paper, $Q / f$ droop control with $K_{Q}=1 e^{-4} \mathrm{~Hz} / \mathrm{VAr}$ is used [12], [29]. The simulation results are depicted in Fig. 8 .

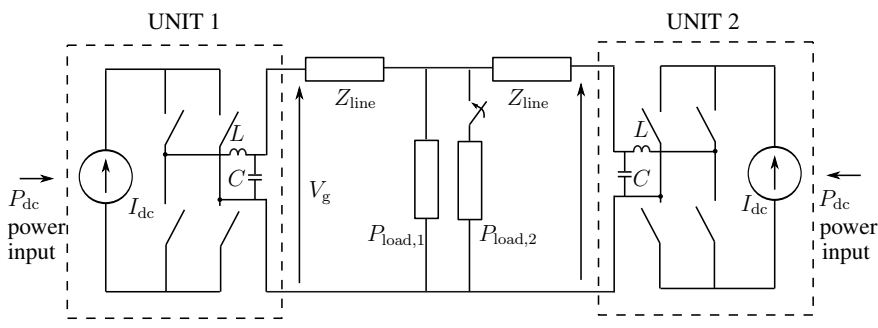

Fig. 7. Configuration: microgrid with two generation units and two constantpower loads

The simulations start from a terminal voltage of $230 \mathrm{~V}$ and $50 \mathrm{~Hz}$ for each DG unit. In the simulations, first a startup transient is shown, with measurements of power only valid after one fundamental period. At $t=1 \mathrm{~s}$, again a small transient is depicted as the second load turns on. In steady-state, with total load 3 p.u., $P_{1}=1.311$ p.u. and

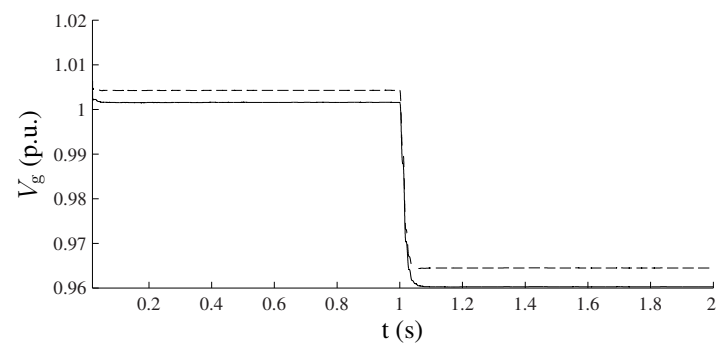

(a) Terminal voltage $V_{g}$

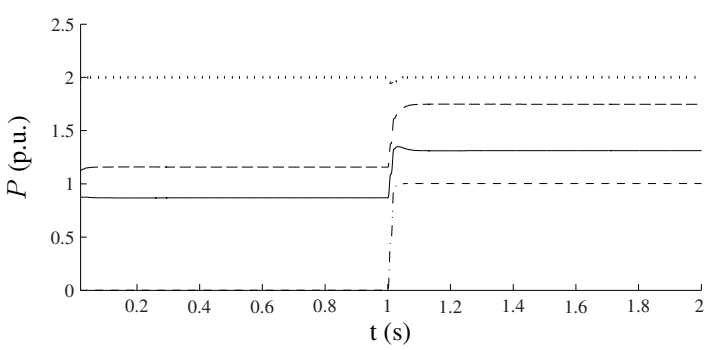

(b) Output power $P$

Fig. 8. GVBD: DG units act on global measure of $V_{\mathrm{dc}}(-=\mathrm{G} 1$; ---- $=\mathrm{G} 2$, $\cdots=\operatorname{load} 1,-.-.-=$ load 2 )

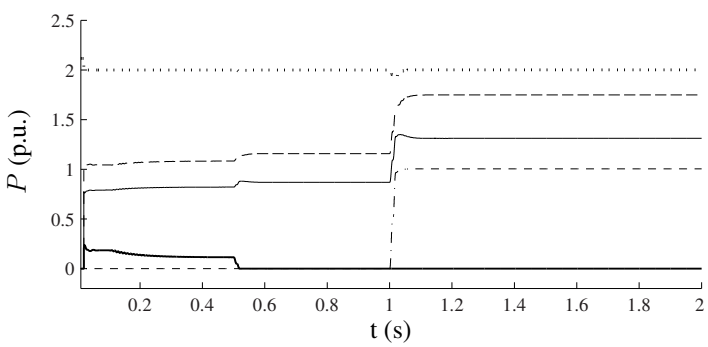

Fig. 9. GVBD with islanding transient,output power $P(-=\mathrm{G} 1 ;----=\mathrm{G} 2$, $\cdots=\operatorname{load} 1, \ldots .-. .=$ load $2,-=$ utility network)

$P_{2}=1.748$ p.u. The power is shared according to the ratings of the DG units because $\frac{P_{1}}{P_{2}}=\frac{P_{1, \text { nom }}}{P_{2, \text { nom }}}=0.75$. The terminal voltages $V_{\mathrm{g}}$ of the units vary proportional to $V_{\mathrm{dc}}$ because of the $V_{\mathrm{g}} / V_{\mathrm{dc}}$ droop controller. In this way, active power changes of renewable energy sources can be postponed compared to that of dispatchable DG units by including constant-power bands based on the local $V_{\mathrm{g}}$, analogous as in the VBD control strategy.

For $0<t<1 \mathrm{~s}, v_{\mathrm{dc}, \mathrm{g}}=\frac{0.435}{V_{\mathrm{dc}, \text { nom }}}$ p.u. This slightly positive value of $v_{\mathrm{dc}, \mathrm{g}}$ implies that $P_{1}+P_{2}<P_{\text {nom }, 1}+P_{\text {nom }, 2}=$ 2.1 p.u. In $1<t<2 \mathrm{~s}, v_{\mathrm{dc}, \mathrm{g}}=\frac{-4.795}{V_{\mathrm{dc} \text { nom }}}$ p.u., which has decreased because of the extra load. Therefore, the generated powers of the DG units increase. In this way, accurate load balancing and load sharing are obtained.

Finally, the performance of this GVBD controller with respect to an islanding transient is studied. Therefore, losing the connection to the upstream grid is simulated. The upstream grid is modelled as a voltage source of $230 \mathrm{~V} / 50 \mathrm{~Hz}$ and is connected to the loads through a 0.009 p.u. line impedance. Fig. 9 shows that despite this large transient, a stable microgrid operation is obtained. The power that was delivered by the utility network in the first $0.5 \mathrm{~s}$, is adequately distributed between the two CIDG units that alter their power delivery after the islanding transient without need for communication.

\section{B. SGs equipped with CPFD control}

The same microgrid is studied, but with SGs instead of DG units. Each SG is represented as an emf $E$ in series with an inductance $L$ (and a small equivalent stator resistance of $0.18 \cdot 10^{-3}$ p.u.), see Fig. 1. The combination of the inductive lines and the SG inductance equals $j 0.028$ p.u. in the simulations. The inertia of the SGs equals $0.18 \mathrm{kgm}^{2}$. A CPFD control is used, i.e. 1 , with $K_{\mathrm{P}, \mathrm{i}}=8000 \pi \frac{P_{\mathrm{i}, \text { nom }}}{P_{1, \text { nom }}+P_{2, \text { nom }}}$. For the reactive power control, $Q / V$ droops are used. In the simulations, by comparing the ac power $P$ with $P_{m}$, the change of frequency is determined through the inertia of the SG. This frequency, together with the obtained rms voltage from the $Q / V$ droops, determine the back-emf $E$ of the SG. The simulation results are depicted in Fig. 10

After a start-up transient and a transient because of the changing load, a stable operation is obtained. As only primary control is implemented, in steady-state $f_{1}=f_{2}$, but not necessarily equal to $f_{\text {nom. }}$. In steady-state, $P_{1}=1.29$ p.u. and $P_{2}=1.71$ p.u., thus, with power sharing according to the ratings of the units. Also in steady state, $P_{1}+P_{2}=$ $P_{\text {load }, 1}=3$ p.u. because the line impedances are assumed as purely inductive, opposed to the previous case. 


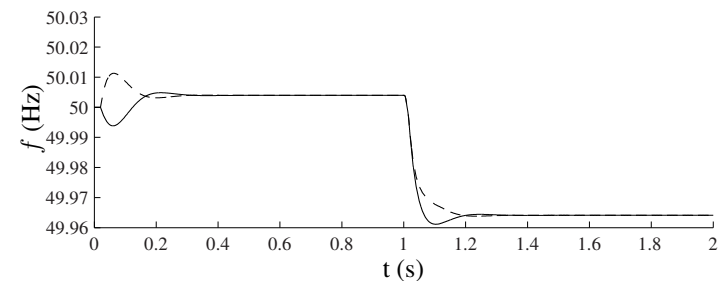

(a) Frequency $f$

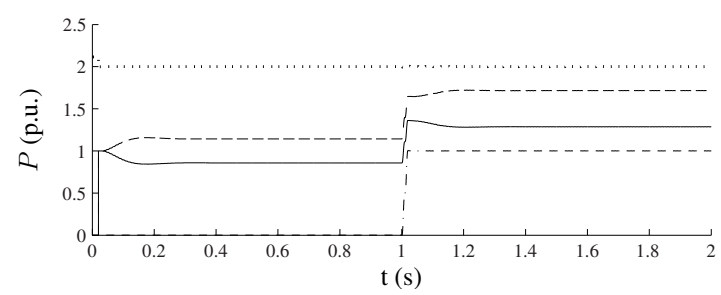

(b) Output power $P$

Fig. 10. SGs equipped with CPFD control, inductive lines $(-=\mathrm{G} 1$; ---- $=$ $\mathrm{G} 2, \cdots=\operatorname{load} 1,-.-\cdot=\operatorname{load} 2$ )

\section{CIDG units with VBD control}

The same case as with the other CIDG units is studied, but with $P / V_{\mathrm{g}}$ droop control without communication instead of $P / v_{\mathrm{dc}, \mathrm{g}}$ droop control. The line parameters are equal to those in the GVBD control scenario. The droop $K_{V}$ of the $P / V_{\mathrm{g}}$ droop controller equals $\sqrt{2} P_{\text {nom }} / 50 \mathrm{~W} / \mathrm{V}$. The results depicted in Fig. 11 show that in steady-state $P_{1}=1.33$ p.u. and $P_{2}=1.74$ p.u. The power is not exactly shared according to the ratings of the units as $P_{1} / P_{2}=0.76$. Exact power sharing is achieved when $R_{\text {line, } 1} / R_{\text {line }, 2}=P_{2} / P_{1}$, then, $P_{1}=1.31$ p.u. and $P_{2}=1.75$ p.u. The power sharing is thus partly dependent on the line parameters. Because of this issue, using the $v_{\mathrm{dc}, \mathrm{g}}$ droop control with communication as secondary control next to the primary voltage-based droop control without communication can give good results. Also, in the scenario of loss of the utility network at $t=0.5 \mathrm{~s}$, a proper operation of the microgrid is obtained, analogous as in the GVBD control as depicted in Fig. 12

In the VBD control, the individual $V_{\mathrm{dc}}$ can vary differently from $v_{\mathrm{dc}, \mathrm{g}}$. This can be advantageous for the line losses in the network. As an example, a microgrid consisting of two zones, with large electrical distances inbetween, is considered. If one zone has a high load burden, in the VBD control, the input powers of the DG units in this zone will increase, while those in the other zones will be little affected. In case the global parameter $v_{\mathrm{dc}, \mathrm{g}}$ is used in the GVBD, both zones will increase their power equally. It is clear that because of the local nature of the VBD control, line losses and congestion problems in the interconnection lines can be reduced.

\section{Microgrid}

In the following simulation, the microgrid case as depicted in Fig. 13 is studied. There are four constant-power loads, with load profile shown in Fig. 14(a), two resistive loads $R_{\text {load,1 }}$ and $R_{\mathrm{load}, 2}$ and three DG units. To limit the simulation time, an averaged converter model is used and the configuration parameters are summarized in Table II. The combination of the line resistances and the resistive virtual output impedances of the DG units $\left(R_{1}, R_{3}\right.$ and $\left.R_{7}\right)$ equals 0.057 p.u.

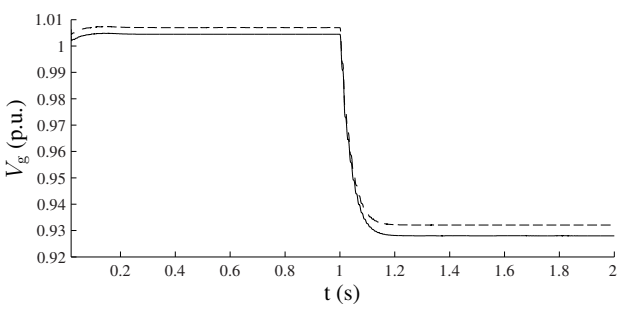

(a) Terminal voltage $V_{\mathrm{g}}$

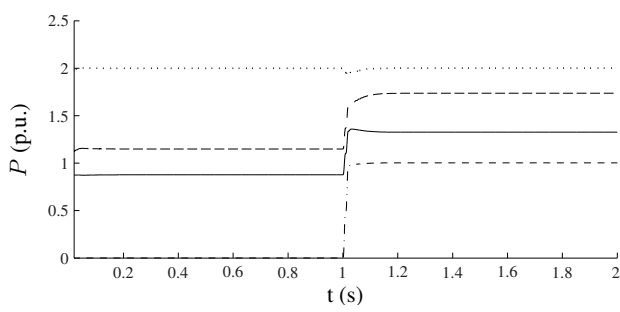

(b) Output power $P$

Fig. 11. VBD: DG units act on local parameters $(-=\mathrm{G} 1 ;----=\mathrm{G} 2,, \cdots$ $=\operatorname{load} 1,-.-.-.=\operatorname{load} 2$ )

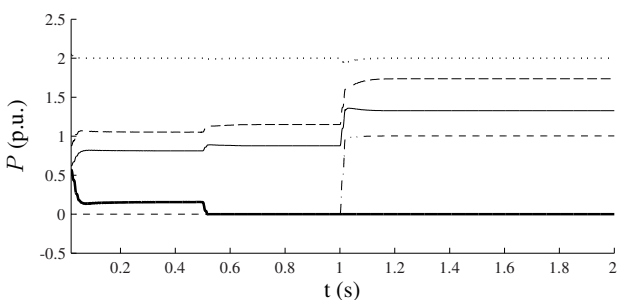

Fig. 12. VBD with islanding transient, output power $P(-=\mathrm{G} 1$; ---- $=\mathrm{G} 2$, $\cdots=\operatorname{load} 1,-.-\cdot=$ load $2,-=$ utility network)

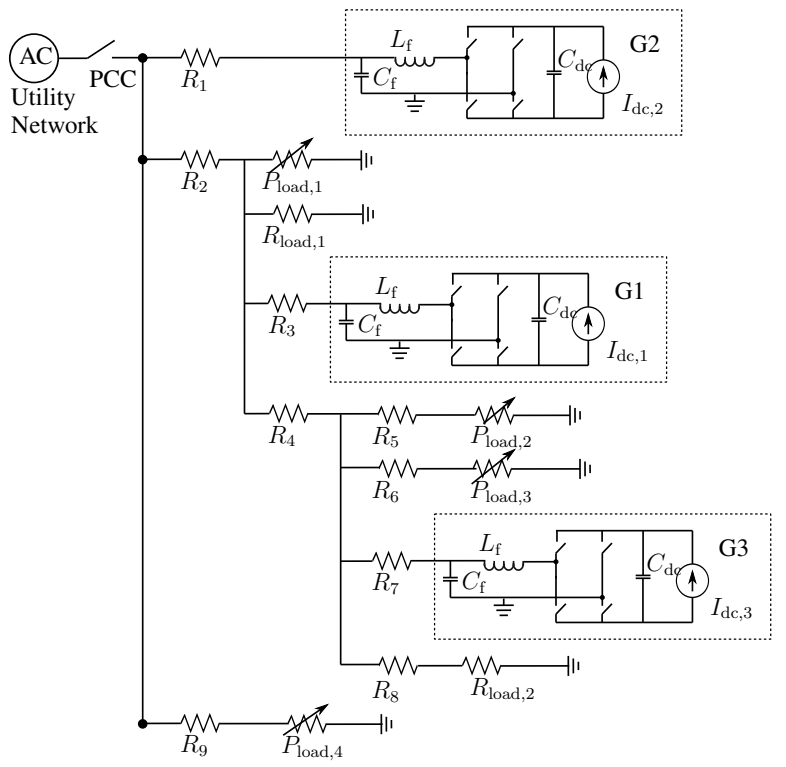

Fig. 13. Islanded microgrid

1) CIDG units with GVBD control: In the first simulation, GVBD control is considered with $v_{\mathrm{dc}, \mathrm{g}}$ calculated from the dc-link voltages of G1 and G2. The third DG unit, G3, does not take part in the power balancing. G3 is considered as a non-dispatchable generator: from $0<t<1 \mathrm{~s}$, G3 operates at 
TABLE II

MICROGRID CASE: PARAMETERS

\begin{tabular}{|c|c|c|c|}
\hline Parameter & value & parameter & value \\
\hline$C_{\mathrm{dc}}$ & $1.5 \mathrm{mF}$ & $R_{\text {load }, 2}$ & 1.42 p.u. \\
\hline$V_{\mathrm{dc}, \mathrm{nom}}$ & $450 \mathrm{~V}$ & $K_{\mathrm{a}}$ & $0.5 / \sqrt{2} \mathrm{~V} / \mathrm{V}$ \\
\hline$V_{\mathrm{g}, \mathrm{ref}}$ & $230 \mathrm{~V}$ & $K_{\mathrm{g}, 1}$ & $90 \frac{P_{\text {nom }, 1}}{P_{\text {nom }, 1+P_{\text {nom }, 2}}} \frac{\mathrm{kW}}{\mathrm{V}}$ \\
\hline$P_{\text {ref }}$ & $1 \mathrm{~kW}$ & $K_{\mathrm{g}, 2}$ & 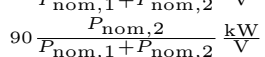 \\
\hline$f_{\text {nom }}$ & $50 \mathrm{~Hz}$ & $K_{\mathrm{g}, 3}$ & $0 \mathrm{~kW} / \mathrm{V}$ \\
\hline$p_{\text {nom }, 1}$ & 2.5 p.u. & $K_{\mathrm{Q}}$ & $0.0001 \mathrm{~Hz} / \mathrm{VAr}$ \\
\hline$p_{\text {nom }, 2}$ & 3 p.u. & $K_{\mathrm{V}, 1}$ & $P_{\mathrm{nom}, 1} / 50 \mathrm{~W} / \mathrm{V}$ \\
\hline$p_{\text {nom }, 3}$ & 2 p.u. & $K_{\mathrm{V}, 2}$ & $P_{\mathrm{nom}, 2} / 50 \mathrm{~W} / \mathrm{V}$ \\
\hline$R_{1}, R_{3}, R_{7}, R_{8}$ & 0.057 p.u. & $K_{\mathrm{V}, 3}$ & $0 \mathrm{~W} / \mathrm{V}$ \\
\hline$R_{2}, R_{4}, R_{5}, R_{6}$ & 0.006 p.u. & $K_{\mathrm{P}, 1}$ & $8 \pi \frac{P_{\text {nom }, 1}}{P_{\text {nom }, 1}+P_{\text {nom }, 2}} \frac{\mathrm{kW}}{\mathrm{Hz}}$ \\
\hline$R_{9}$ & 0.006 p.u. & $K_{\mathrm{P}, 2}$ & 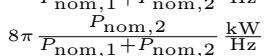 \\
\hline$R_{\text {load }, 1}$ & 0.95 p.u. & $K_{\mathrm{P}, 3}$ & 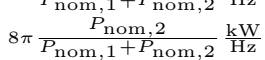 \\
\hline
\end{tabular}

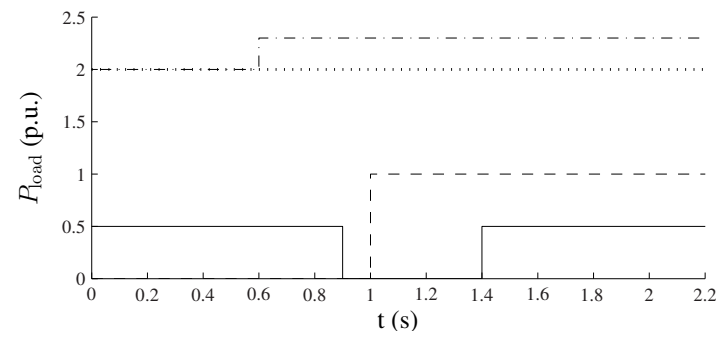

(a) Constant-Power loads $\left(-=P_{\text {load }, 1} ;----=P_{\text {load }, 2},-.-.-.=\right.$ $\left.P_{\text {load }, 3}, \cdots=P_{\text {load }, 4}\right)$

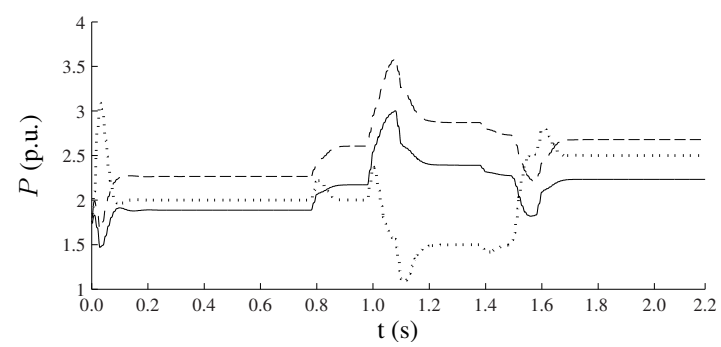

(b) Output power $P$

Fig. 14. Microgrids with DG units with GVBD control $(-=\mathrm{G} 1$; ---- $=\mathrm{G} 2$, $\cdots=\mathrm{G} 3$

$P_{\text {nom }, 3}$, from $1<t<1.5 \mathrm{~s}$, the generated power decreases with $25 \%$; from then on, the generated power increases again to $1.25 P_{\text {nom }, 3}$. Next to the $P / v_{\mathrm{dc}, \mathrm{g}}$ droops with slope $K_{\mathrm{g}}$, also the $V_{\mathrm{g}} / V_{\mathrm{dc}}$ droop with slope $K_{\mathrm{a}}$ and $Q / f$ droops with slope $K_{\mathrm{Q}}$ are used. The results are depicted in Fig. 14

After a start-up transient, a stable operation is obtained. The undispatchable DG unit G3 delivers its nominal power from 0 to $1 \mathrm{~s}$, despite the load changes. This unit does not take part in the power sharing according to the ratings. The other two DG units take part in this power sharing and power balancing acting on the load changes. For example, in steady-state, $P_{1}=2.23$ p.u., $P_{2}=2.68$ p.u., thus, $P_{1} / P_{2}=P_{\text {nom }, 1} / P_{\text {nom }, 2}=0.83$. Therefore, the GVBD control works well, showing that making an analogy between CPFD control and microgrid control with resistive line parameters is possible, but communication is required.

2) SGs with CPFD control: In the analogous CPFD control, instead of DG units, the microgrid is powered by directly

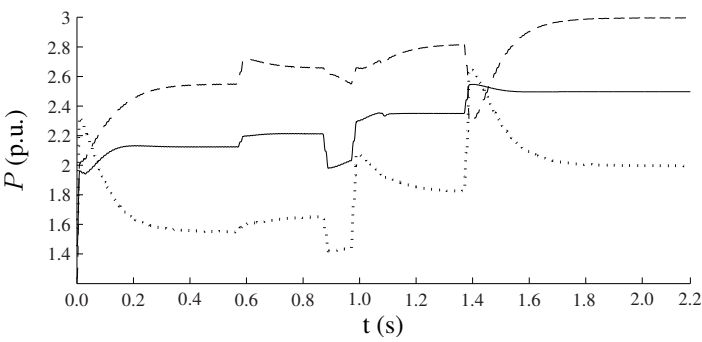

Fig. 15. Microgrids with SGs controlled by CPFD control, output power $P$ $(-=\mathrm{G} 1 ;----\mathrm{G} 2, \cdots=\mathrm{G} 3)$

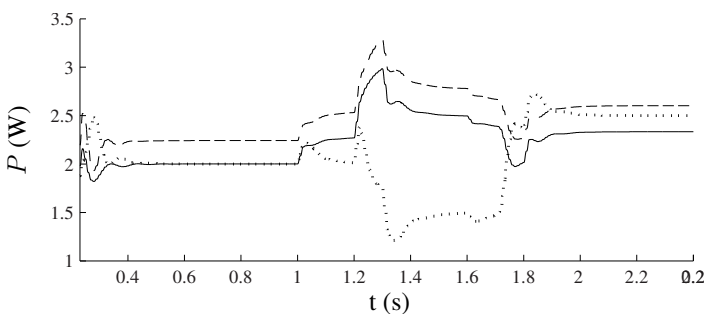

Fig. 16. Microgrids with DG units controlled by VBD control, output power $P(-=\mathrm{G} 1 ;---=\mathrm{G} 2, \cdots=\mathrm{G} 3)$

coupled SGs. The three units are equipped with $P / f$ droop control to take part in the power balancing as most SGs are dispatchable opposed to many small DG units. As the networks powered by SGs are generally high-voltage networks, the lines here are considered as mainly inductive. Except for this, the considered microgrid is the same as in the previous case. Next to the $P / f$ droops with slope $K_{\mathrm{P}}$, also $Q / V$ droops with slope $0.01 / \sqrt{2}$ V/VAr are used. The results are depicted in Fig. 15

For example, at $t=2.20 \mathrm{~s}, P_{1}=2.50$ p.u., $P_{2}=3.00$ p.u. and $P_{3}=2.00$ p.u.; while at $t=1.4 \mathrm{~s}, P_{1}=2.35$ p.u., $P_{2}=2.81$ p.u. and $P_{3}=1.82$ p.u. In steady-state, the power is shared according to the ratings of the SGs without communication. After each transient, the frequency measured by the three units may differ, but only slightly, thus only phase angle differences are obtained. In steady-state, the frequencies of the units are equal as frequency is a global parameter. The steady-state frequency is not necessarily equal to $50 \mathrm{~Hz}$ as only primary control is considered in this network.

3) CIDG units with VBD control: The same microgrid configuration as with the GVBD control is studied, but with VBD control. Next to the $P / V_{\mathrm{g}}$ droops with slope $K_{\mathrm{V}}$, also the $V_{\mathrm{g}} / V_{\mathrm{dc}}$ droops with slope $K_{\mathrm{a}}$ and $Q / f$ droops with slope $K_{\mathrm{Q}}$ are used. The results are depicted in Fig. 16

After a start-up transient, a stable operation is obtained. In steady-state at $t=2.2 \mathrm{~s}, P_{3}=2.5$ p.u. as this is the undispatchable DG unit; $P_{1}=2.3$ p.u., $P_{2}=2.6$ p.u., thus, $P_{1} / P_{2}=0.89$. This illustrates a well-known characteristic of droop control without communication, namely the inherent trade-off between accuracy of power sharing and voltage deviations [23], [24], [30]. It also shows that the GVBD control can help to optimize the power sharing according to the ratings of the units, e.g., when included as a secondary control strategy, requiring communication. VBD control is used as primary control because of reliability reasons as it does not depend on communication links to achieve a proper microgrid operation. 


\section{CONCLUSiOnS}

In this paper, the CPFD control in inductive networks based on the rotating inertia of the SGs and the control of resistive microgrids by means of CIDG units lacking inertia, are compared. The theoretical analogy between the rotating inertia and the dc-link capacitor on one hand and the grid frequency and the dc-link voltage on the other hand are studied. A control strategy based on this analogy is presented, which is called the GVBD control. Opposed to CPFD control, the GVBD control requires inter-unit communication to exploit the analogy with conventional networks. This is because the dc-link voltage is not a global parameter, opposed to grid frequency. With the GVBD control, accurate power sharing, a stable operation, and an operation similar to conventional grid control are obtained.

The VBD control is based on the same principles as the GVBD control but without inter-unit communication, which benefits the reliability of the system. Therefore, it is a control strategy for resistive islanded microgrids that approaches the analogy with CPFD control closely, so an operation similar to that of the conventional network can be obtained. The local nature of this control strategy can lead to a possible reduction of line losses and congestion problems. It can also lead to an optimized integration of renewable energy sources in the network because of the usage of constant-power bands with a width depending on the nature of the energy source. It is also discussed in this paper that the power sharing can be optimized by using the VBD control as a primary control strategy with GVBD control, or a variant of this, as secondary controller.

\section{REFERENCES}

[1] J. A. Peças Lopes, N. Hatziargyriou, J. Mutale, P. Djapic, and N. Jenkins, "Integrating distributed generation into electric power systems: A review of drivers, challenges and opportunities," Electric Power Systems Research, vol. 77, pp. 1189-1203, 2007.

[2] N. Jenkins, R. Allan, P. Crossley, D. Kirschen, and G. Strbac, Embedded Generation, ser. IEE Power and Energy systems. The Institution of Electrical Engineers, Oct. 2000, vol. 31.

[3] A.Piccolo and P. Siano, "Evaluating the impact of network investment deferral on distributed generation expansion," IEEE Trans. Power Syst., vol. 24, no. 3, pp. 1559-1567, Aug. 2009.

[4] R. Lasseter, "Microgrids," Proc. Power Eng. Soc. Winter Meeting, pp. 305-308, 2002.

[5] R. H. Lasseter, A. Akhil, C. Marnay, J. Stephens, J. Dagle, R. Guttromson, A. Meliopoulous, R. Yinger, and J. Eto, "The CERTS microgrid concept, white paper on integration of distributed energy resources," in California Energy Commission, Office of Power Technologies - U.S. Department of Energy, LBNL-50829, http://certs.lbl.gov, Apr. 2002.

[6] A. Engler, O. Osika, M. Barnes, N. Jenkins, and A. Arulampalam, DB1 Local Micro Source controller strategies and algorithms. www.microgrids.eu/micro2000, European Commission, Feb. 2004.

[7] R. H. Lasseter, J. H. Eto, B. Schenkman, J. Stevens, H. Vollkommer, D. Klapp, E. Linton, H. Hurtado, and J. Roy, "CERTS microgrid laboratory test bed," IEEE Trans. Power Del., vol. 26, no. 1, pp. 325332, Jan. 2011.

[8] T. C. Green and M. Prodanovíc, "Control of inverter-based micro-grids," Electric Power Systems Research, vol. 77, no. 9, pp. 1204-1213, July 2007.

[9] M. C. Chandorkar, D. M. Divan, and R. Adapa, "Control of parallel connected inverters in standalone ac supply systems," IEEE Trans. Ind. Appl., vol. 29, no. 1, pp. 136-143, Jan./Feb. 1993.

[10] J. M. Guerrero, J. Matas, L. García de Vicuña, M. Castilla, and J. Miret, "Wireless-control strategy for parallel operation of distributed-generation inverters," IEEE Trans. Ind. Electron., vol. 53, no. 5, pp. 1461-1470, Oct. 2006.

[11] J. A. Peças Lopes, C. L. Moreira, and A. G. Madureira, "Defining control strategies for microgrids in islanded operation," IEEE Trans. Power Syst., vol. 21 , no. 2, pp. 916-924, 2006.
[12] H. Laaksonen, P. Saari, and R. Komulainen, "Voltage and frequency control of inverter based weak LV network microgrid," in 2005 International Conference on Future Power Systems, Amsterdam, Nov. 18, 2005.

[13] A. Engler, O. Osika, M. Barnes, and N. Hatziargyriou, DB2 Evaluation of the local controller strategies. www.microgrids.eu/micro2000, Jan. 2005.

[14] T. L. Vandoorn, B. Meersman, L. Degroote, B. Renders, and L. Vandevelde, "A control strategy for islanded microgrids with dc-link voltage control," IEEE Trans. Power Del., vol. 26, no. 2, pp. 703-713, Apr. 2011.

[15] J. M. Guerrero, J. Matas, L. García de Vicuña, M. Castilla, and J. Miret, "Decentralized control for parallel operation of distributed generation inverters using resistive output impedance," IEEE Trans. Ind. Electron., vol. 54, no. 2, pp. 994-1004, Apr. 2007.

[16] J. Matas, L. G. de Vicuña, and J. C. Vasquez, "Virtual impedance loop for droop-controlled single-phase parallel inverters using a second order general integrator scheme," IEEE Trans. Power Electron., vol. 25, no. 12, p. 2993, Dec. 2010.

[17] T. L. Vandoorn, B. Renders, L. Degroote, B. Meersman, and L. Vandevelde, "Active load control in islanded microgrids based on the grid voltage," IEEE Trans. on Smart Grid, vol. 2, no. 1, pp. 139-151, Mar. 2011.

[18] W. Yao, M. Chen, J. M. Guerrero, and Z.-M. Qian, "Design and analysis of the droop control method for parallel inverters considering the impact of the complex impedance on the power sharing," IEEE Trans. Ind. Electron., vol. 58, no. 2, pp. 576-588, Feb. 2011.

[19] M. Marwali, J.-W. Jung, and A. Keyhani, "Control of distributed generation systems - part II: Load sharing control," IEEE Trans. Power Electron., vol. 19, no. 6, pp. 1551-1561, Nov. 2004.

[20] F. Katiraei, R. Iravani, N. Hatziargyriou, and A. Dimeas, "Microgrids management: controls and operation aspects of microgrids," Power and Energy Magazine, vol. 6, no. 3, pp. 54-65, May/June 2008.

[21] K. Debrabandere, B. Bolsens, J. Van den Keybus, A. Woyte, J. Driesen, and R. Belmans, "A voltage and frequency droop control method for parallel inverters," IEEE Trans. Power Electron., vol. 22, no. 4, pp. $1107-1115$, July 2007.

[22] F. A. Bhuiyan and A. Yazdani, "Multimode control of a DFIG-Based wind-power unit for remote applications," IEEE Trans. Power Del., vol. 24, no. 4, pp. 2079-2089, Oct. 2009.

[23] J. M. Guerrero, L. García de Vicuña, J. Matas, M. Castilla, and J. Miret, "Output impedance design of parallel-connected UPS inverters with wireless load-sharing control," IEEE Trans. Ind. Electron., vol. 52, no. 4, pp. 1126-1135, Aug. 2005.

[24] J. M. Guerrero, L. Hang, and J. Uceda, "Control of distributed uninterruptible power supply systems," IEEE Trans. Ind. Electron., vol. 55, no. 8, pp. 2845-2859, Aug. 2008.

[25] A. Engler, "Applicability of droops in low voltage grids," DER Journal, no. 1, Jan. 2005.

[26] A. Engler and N. Soultanis, "Droop control in LV-grids," in Proc. Internat. Conf. On Future Power Systems, The Netherlands, Nov. 16$18,2005$.

[27] C. Sao and P. Lehn, "Control and power management of converter fed microgrids," IEEE Trans. Power Syst., vol. 23, no. 3, Aug. 2008.

[28] M. Bollen, J. Zhong, O. Samuelsson, and J. Björnstedt, "Performance indicators for microgrids during grid-connected and island operation," in Proc. IEEE Power Tech. Conf., Bucharest, Romania, June 28 - July 2, 2009.

[29] T. L. Vandoorn, B. Renders, B. Meersman, L. Degroote, and L. Vandevelde, "Reactive power sharing in an islanded microgrid," in 45th International Universities Power Engineering Conference (UPEC 2010), Cardiff, Wales, Aug. 31 - Sep. 3, 2010.

[30] A. Tuladhar, H. Jin, T. Unger, and K. Mauch, "Control of parallel inverters in distributed $\mathrm{AC}$ power systems with consideration of line impedance effect," IEEE Trans. Ind. Appl., vol. 36, no. 1, pp. 131-138, Jan./Feb. 2000. 


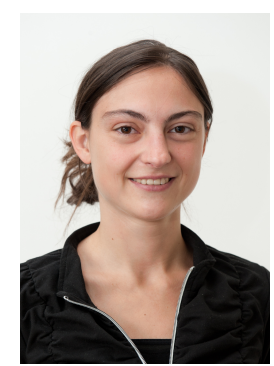

Tine Vandoorn (S'09) was born in Torhout, Belgium in 1985. She received the M.S. in electromechanical engineering from Ghent University, Ghent, Belgium, in 2008. In 2008, she joined the Electrical Energy Laboratory (EELAB) of Ghent University where she is currently pursuing the Ph.D. degree. Her present research interests include electric power systems, voltage and power control of DG units, management of microgrids and smart microgrids. In 2009, she was awarded a grant as Ph.D. fellow of the Research Foundation Flanders (FWO).

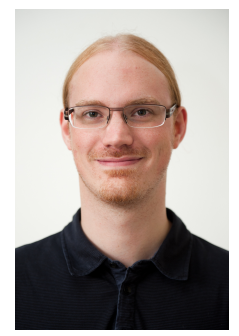

Jeroen De Kooning ( $S^{\prime}$ 09) was born in Kapellen, Belgium in 1987. He received the M.S. degree in electromechanical engineering from Ghent University, Belgium, in 2010. Since then, he is with the Electrical Energy Laboratory (EELAB) of Ghent University and is currently pursuing the Ph.D degree. His present research interests include wind energy systems and control of power electronic converters.

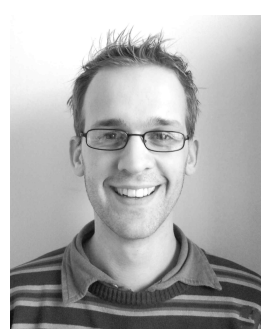

Bart Meersman (S'07) was born in Sint-Niklaas, Belgium on July 29, 1983. He received the M.S degree in electromechanical engineering from Ghent University, Belgium, in 2006. Since then, he is with the Electrical Energy Laboratory (EELAB) of Ghent University and is currently working towards a $\mathrm{Ph} . \mathrm{D}$. degree. His present research interests include dynamic phasors, renewable energy applications, digital control of power electronic converters and their contribution to power quality.

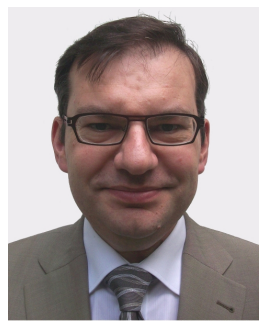

Lieven Vandevelde (M'05 - SM'07) was born in Eeklo, Belgium, in 1968. He graduated as Master of Electromechanical Engineering and received the Ph.D. degree from Ghent University, Belgium, in 1992 and 1997, respectively. He is with the Electrical Energy Laboratory (EELAB), Ghent University, where he has been a Professor in electrical power engineering since 2004. His research and teaching activities are in the field of electric power systems, electrical machines and (computational) electromagnetics. 